\title{
ASYMPTOTIC PERIODICITY OF SOLUTIONS TO A CLASS OF NEUTRAL FUNCTIONAL DIFFERENTIAL EQUATIONS
}

\author{
JIANHONG WU
}

(Communicated by Kenneth R. Meyer)

\begin{abstract}
In this paper, we extend a convergence result due to Takáč to continuous maps satisfying certain monotonicity properties. Applying this extension to the Poincare map associated with the neutral equation

$$
(d / d t)[x(t)-b(t) x(t-r)]=F[t, x(t), x(t-r)]
$$

we prove that each solution of the above neutral equation tends to an $r$-periodic function as $t \rightarrow \infty$ in an oscillatory manner, where $0 \leq b(t)<1$ is an $r$ periodic continuous function and $F$ satisfies a certain order relation.
\end{abstract}

\section{INTRODUCTION}

In [9], it is shown that each solution of the scalar neutral functional differential equation

$$
\frac{d}{d t}[x(t)-b x(t-r)]=F[t, x(t), x(t-r)], \quad t \geq 0, r \geq 0,
$$

approaches a constant as $t \rightarrow \infty$, where $0 \leq b<1$ is a constant and $F$ satisfies the order relation $F(t, x, y) \leq 0$ if $x \geq y$, and $F(t, x, y) \geq 0$ if $x \leq y$. This indicates an asymptotic equivalence between the solution of neutral equation (1.1) and the solution of the ordinary differential equation $(d / d t)[x(t)-b x(t)]=0$.

The major purpose of this paper is to extend this result to the following scalar periodic neutral functional differential equation

$$
\frac{d}{d t}[x(t)-b(t) x(t-r)]=F[t, x(t), x(t-r)], \quad t \geq 0
$$

where $0 \leq b(t)<1$ is an $r$-periodic continuous function and $F(t, x, y)$ is $r$-periodic in $t$ and satisfies the above order relation. Since each solution of the ordinary differential equation $(d / d t)[(1-b(t)) x(t)]=0$ is $r$ periodic and can be expressed as $x(t)=((1-b(0)) /(1-b(t))) x(0)$, it is natural

Received by the editors October 19, 1989 and, in revised form, December 20, 1989.

1980 Mathematics Subject Classification (1985 Revision). Primary 34K20, 34K25.

Key words and phrases. Asymptotic periodicity, neutral equations, monotone systems.

Research supported by Gordin Kaplan Award Postdoctoral Fellowship. 
to conjecture that any solution of (1.2) tends to a multiple of $1 /(1-b(t))$ as $t \rightarrow \infty$.

Much research has been devoted to the study of the asymptotic behavior of solutions for (1.2) with $b(t) \equiv 0$ (see, e.g., [1-8, 13]) or with a constant $b \in[0,1)$ (see, e.g., [9, 17]). Various approaches such as the first integral, Liapunov function coupled with the Razumikhin technique, invariance principle, etc. have been applied to investigate the problem of convergence of solutions. In this paper, we take a rather different point of view in dealing with this problem. Indeed, we investigate the relation between the convergence of solutions and the monotonicity of the associated Poincare map as well as the structure of its fixed point set. We will show that the associated Poincare map enjoys certain monotonicity properties, its set of fixed points contains a straight line in a positive direction, and each solution of (1.2) approaches a multiple of $1 /(1-b(t))$ in an oscillatory manner.

This paper is organized as follows. In $\S 2$ we extend a convergence result due to Takác [16] for strong monotone maps to a continuous map satisfying certain weak monotonicity properties described in assumptions (i) and (ii) of Theorem 2.1. In $\S 3$ we employ the technique in [9] and [15] to associate neutral equation (1.2) with a retarded equation with unbounded delay in order to verify the boundedness of solutions and the monotonicity of the associated Poincare map of (1.2). Applying the general results from $\S 2$ we prove that each solution of (1.2) approaches a periodic function and the convergence occurs in an oscillatory manner.

\section{A GENERAL CONVERGENCE RESULT}

Let $X$ denote a strongly ordered space, i.e., a metrizable topological space together with a closed partial order relation $R \subseteq X \times X$ such that Int $R \neq \varnothing$. For any $x, y, q \in X$ and any subset $A \subseteq X$, the following notations will be employed: $x \leq y$ iff $(x, y) \in R, x<y$ if $(x, y) \in R$ and $x \neq y, x \ll y$ iff $(x, y) \in \operatorname{Int} R, A \ll q$ iff $a \ll q$ for $a \in A, q \ll A$ iff $q \ll a$ for $a \in A$, $A \leq q$ iff $a \leq q$ for $a \in A, q \leq A$ iff $q \leq a$ for $a \in A$.

We consider a continuous map $S: X \rightarrow X$. Let $E$ denote the set of all fixed points of $S$, i.e., $E=\{e \in X ; S(e)=e\}$. For any given $e \in E$, we define $S^{e}=\{x \in X ; e \leq x\}$ and $S_{e}=\{x \in X ; x \leq e\}$.

The main result in this section is as follows:

Theorem 2.1. Suppose that

(i) for any $e \in E, S\left(S^{e}\right) \subseteq S^{e}$ and $S\left(S_{e}\right) \subseteq S_{e}$;

(ii) for any $e \in E$, there exists an integer $N \geq 1$ such that $e \ll S^{n}\left(S^{e} \backslash\{e\}\right)$ and $S^{n}\left(S_{e} \backslash\{e\}\right) \ll e$ for all $n \geq N$;

(iii) $E$ contains a simply ordered arc given by $J: R \rightarrow X$ (i.e., $J: \mathbb{R} \rightarrow X$ is continuous and $\tau_{1}<\tau_{2}$ implies $J\left(\tau_{1}\right)<J\left(\tau_{2}\right)$ ) such that for every $x \in X$ there exist $\alpha, \beta \in \mathbb{R}$ with $J(\alpha) \leq x \leq J(\beta)$. 
Then if $x \in X$ is given such that its semiorbit $0^{+}(x):=\left\{S^{n}(x) ; n=0,1,2, \ldots\right\}$ has compact closure, then its $\omega$-limit set $\omega(x):=\bigcap_{j \geq 0} \mathrm{cl}_{n \geq j} S^{n}(x)$ is a single equilibrium and $\omega(x) \subseteq J(\mathbb{R})$.

Proof. Let $x \in X$ be given such that $0^{+}(x)$ has compact closure. Then by assumption (iii) there exist $\alpha, \beta \in \mathbb{R}$ with $J(\alpha) \leq x \leq J(\beta)$. Since $J(\mathbb{R}) \subseteq E$, by assumption (i) we get $J(\alpha) \leq S^{n}(x) \leq J(\beta)$ for nonnegative integer $n$. This implies that $J(\alpha) \leq \omega(x) \leq J(\beta)$.

Define

$$
\alpha^{*}=\sup \{\alpha \in \mathbb{R} ; J(\alpha) \leq \omega(x)\}, \quad \beta^{*}=\inf \{\beta \in \mathbb{R} ; \omega(x) \leq J(\beta)\} .
$$

Then $J\left(\alpha^{*}\right) \leq \omega(x) \leq J\left(\beta^{*}\right)$. We want to show that $\alpha^{*}=\beta^{*}$. Suppose not, i.e., $\alpha^{*}<\beta^{*}$. Then $J\left(\alpha^{*}\right)<J\left(\beta^{*}\right)$. Since $J\left(\alpha^{*}\right)$ and $J\left(\beta^{*}\right)$ are fixed points of $S$, by assumption (ii) we get $J\left(\alpha^{*}\right) \ll J\left(\beta^{*}\right)$. Therefore, we can find a neighborhood $N\left(\alpha^{*}\right)$ of $J\left(\alpha^{*}\right)$ and a neighborhood $N\left(\beta^{*}\right)$ of $J\left(\beta^{*}\right)$ such that $y \ll z$ for $(y, z) \in N\left(\alpha^{*}\right) \times N\left(\beta^{*}\right)$.

If $J\left(\alpha^{*}\right) \in \omega(x)$, then there exists a positive integer $m$ such that $S^{m}(x) \in$ $N\left(\alpha^{*}\right)$, and thus $S^{m}(x) \ll J\left(\beta^{*}\right)$. Because of the continuity and the increasing property of $J$, we can find a real number $\gamma^{*}<\beta^{*}$ such that $S^{m}(x) \leq J\left(\gamma^{*}\right) \ll$ $J\left(\beta^{*}\right)$. Applying assumption (i), we obtain $S^{n}(x) \leq J\left(\gamma^{*}\right) \ll J\left(\beta^{*}\right)$ for all $n \geq m$. This implies that $\omega(x) \leq J\left(\gamma^{*}\right) \ll J\left(\beta^{*}\right)$, a contradiction to the definition of $\beta^{*}$.

Therefore if $\alpha^{*} \neq \beta^{*}$, then $J\left(\alpha^{*}\right) \notin \omega(x)$, i.e., $J\left(\alpha^{*}\right)<q$ for all $q \in \omega(x)$. By assumption (ii) and the invariance of $\omega(x)$, we obtain $J\left(\alpha^{*}\right) \ll \omega(x)$. But this contradicts the definition of $\alpha^{*}$. Therefore, $\alpha^{*}=\beta^{*}$ and $\omega(x)=J\left(\alpha^{*}\right) \epsilon$ $E$. The proof is completed.

Remark 2.1. We recall that a continuous map $S: X \rightarrow X$ is monotone if $x \leq y$ implies $S(x) \leq S(y)$ for all $x, y \in X$; eventually strongly monotone if $x<y$ implies $S^{n}(x) \ll S^{n}(y)$ for all $x, y \in X$ and $n \geq N$, where $N \geq 1$ is an integer independent of $x$ and $y$; strongly monotone if $x<y$ implies $S^{n}(x) \ll S^{n}(y)$ for all $x, y \in X$ and $n \geq 1$ (see, e.g., [11] and [16]). Evidently, if $S$ is monotone, then the assumption (i) is satisfied, and if $S$ is eventually strongly monotone, then the assumption (ii) is satisfied. However, we should emphasize that assumptions (i) and (ii) only require the map $S$ preserve the order relation between two points in $X$ such that one of them is an equilibrium. Usually, $E$ is very small compared to the whole space. Consequently, the verification of assumptions (i) and (ii) is easier than that of the monotonicity and the eventual strong monotonicity of $S$. In particular, for system (1.2) we will show that the associated Poincaré map satisfies assumptions (i) and (ii), but we are unable to verify the eventual strong monotonicity of the Poincare map.

To conclude this section, we should mention that Theorem 2.1 was proved by Takác in [16, Theorem 1.3] under the assumption that $S$ is strongly monotone. Our proof is similar to Takáć's proof. However, to replace the strong monotonicity by the weak assumptions (i) and (ii), certain modification of Takáč's proof has to be made, because Takáč used the fact that for a strongly monotone 
map the $\omega$-limit set of a relative compact semiorbit is unordered, but we do not know if this fact is still true or not for a continuous map satisfying assumptions (i) and (ii).

\section{APPLICATIONS TO THE ASYMPTOTIC PERIODICITY PROBLEM FOR NEUTRAL EQUATIONS}

Applications of the general result from $\S 2$ to differential equations require the verification of the following conditions:

(i) the monotonicity of solutions described by assumption (i);

(ii) the strong monotonicity of solutions described by assumption (ii);

(iii) the structure of the fixed point set described by assumption (iii);

(iv) relative compactness of solutions.

In this section, we show that the above verification can be accomplished for (1.2) by associating neutral equation (1.2) with a retarded equation with unbounded delay and by using the Liapunov-Razumikhin technique.

We consider the following scalar neutral functional differential equation

$$
\frac{d}{d t}[x(t)-b(t) x(t-r)]=F[t, x(t), x(t-r)], \quad r>0,
$$

where $b(t)$ is a continuous $r$-periodic function and

(a1) $0 \leq b(t)<1$ for $t \in(-\infty,+\infty)$;

(a2) $F(t, x, y)$ is continuous in $(t, x, y) \in R^{3}$, and $r$-periodic in $t$;

(a3) $F(t, x, x)=0$ for all $(t, x) \in R^{2}$;

(a4) $F(t, x, y)$ is increasing in $y$ when $(t, x) \in R^{2}$ is fixed;

(a5) for any bounded set $W \subseteq R^{3}$ there exists a constant $L>0$ such that

$$
F(t, x, y) \geq-L(x-y) \text { for any }(t, x, y) \in W .
$$

Equation (3.1) has been used in the study of classical electron radiation, epidemics, population growth, and biological compartmental systems. The convergence to constant functions as $t \rightarrow \infty$ of the solution of (3.1) in the case where $b$ is a constant has been investigated in $[1-9,13]$. The purpose of this section is to show the convergence to $r$-periodic functions as $t \rightarrow \infty$ of the solution of (3.1) in the case where $b$ is $r$-periodic.

Define $C=C\left([-r, 0], \mathbb{R}^{1}\right)$ as the Banach space of continuous functions on $[-r, 0]$ with a supremum norm. We introduce the following order relation $R \subseteq C \times C$ :

$$
\begin{aligned}
& (\varphi, \psi) \in R \text { iff } \varphi(\theta) \leq \psi(\theta) \text { for } \theta \in[-r, 0] \text { and } \\
& \varphi(0)-b(0) \varphi(-r) \leq \psi(0)-b(0) \psi(-r) .
\end{aligned}
$$

Evidently, $R$ is a closed-order relation on $C$ and Int $R \neq \varnothing$. Therefore, $C$ endowed with the above-order relation is a strongly ordered space.

For simplification, we define the map $D: \mathbb{R}^{1} \times C \rightarrow \mathbb{R}^{1}$ by $D(t, \varphi)=$ $\varphi(0)-b(t) \varphi(-r)$ for $(t, \varphi) \in \mathbb{R}^{1} \times C$. Moreover for any continuous function $x:[-r, \infty) \rightarrow \mathbb{R}^{1}$ and $t \geq 0$, we define $x_{t} \in C$ by $x_{t}(\theta)=x(t+\theta)$ for $\theta \in[-r, 0]$. 
Denoting the solution of (3.1) satisfying $x_{0}=\varphi$ by $x(t, \varphi)$, we have the following result:

Lemma 3.1. For any $\varphi \in C$, we have

$$
\begin{aligned}
\frac{1}{1-b(t)} m(\varphi) & \leq x(t, \varphi) \leq \frac{1}{1-b(t)} M(\varphi), \\
m(\varphi) & \leq D\left(t, x_{t}(\varphi)\right) \leq M(\varphi)
\end{aligned}
$$

for $t \geq 0$, where

$$
\begin{aligned}
& m(\varphi)=\min \left\{\min _{s \in[-r, 0]}[1-b(s)] \min _{\tau \in[-r, 0]} \varphi(\tau), D(0, \varphi)\right\}, \\
& M(\varphi)=\max \left\{\max _{s \in[-r, 0]}[1-b(s)] \max _{\tau \in[-r, 0]} \varphi(\tau), D(0, \varphi)\right\} .
\end{aligned}
$$

Proof. Let $x(t)=x(t, \varphi)$ and $y(t)=D\left(t, x_{t}\right)$. Then, since $b$ is $r$-periodic,

$$
x(t)=\sum_{i=0}^{[t / r]} b^{i}(t) y(t-i r)+b^{[t / r]+1}(t) x\left(t-\left(\left[\frac{t}{r}\right]+1\right) r\right)
$$

from where it follows that

$$
x(t-r)=\sum_{i=1}^{[t / r]} b^{i-1}(t) y(t-i r)+b^{[t / r]}(t) x\left(t-\left(\left[\frac{t}{r}\right]+1\right) r\right) .
$$

Clearly, $y(0) \leq M(\varphi)$. On the other hand, at any instant where $\tau \geq 0$ such that $y(\tau)=\max _{0 \leq s \leq \tau} y(s)$ and $y(\tau) \geq M(\varphi)$, we have

$$
\begin{aligned}
x(\tau- & r)[1-b(\tau)] \\
& =\left\{\sum_{i=1}^{[\tau / r]} b^{i-1}(\tau) y(\tau-i r)+b^{[\tau / r]}(\tau) x\left(\tau-\left(\left[\frac{\tau}{r}\right]+1\right) r\right)\right\}[1-b(\tau)] \\
& \leq\left[\sum_{i=1}^{[\tau / r]} b^{i-1}(\tau)+b^{[\tau / r]}(\tau) \cdot \frac{1}{1-b(\tau)}\right] y(\tau) \cdot[1-b(\tau)] \\
& =y(\tau),
\end{aligned}
$$

and thus

$$
x(\tau)-x(\tau-r)=y(\tau)-(1-b(\tau)) x(\tau-r) \geq 0,
$$

from which and by assumptions (a3) and (a4), we obtain $\dot{y}(\tau) \leq 0$. Therefore, $y(t) \leq M(\varphi)$ for all $t \geq 0$. On the other hand, by the definition of $M(\varphi)$, $x\left(t-\left(\left[\frac{t}{r}\right]+1\right) r\right) \leq M(\varphi) /(1-b(t))$ for $t \geq 0$. Therefore using (3.2) we obtain $x(t) \leq M(\varphi) /(1-b(t))$. That is, we have shown that $x(t, \varphi) \leq M(\varphi) /(1-b(t))$ and $D\left(t, x_{t}(\varphi)\right) \leq M(\varphi)$ for $t \geq 0$. The other part of the conclusion can be proved analogously.

Lemma 3.2. Let $x(t, \varphi)$ denote an r-periodic solution of $(3.1)$, and $x(t, \psi)$ be any given solution of (3.1). Then

(i) $\varphi \leq \psi$ implies that $x_{t}(\varphi) \leq x_{t}(\psi)$ for all $t \geq 0$; 
(ii) $\varphi<\psi$ implies that $x_{t}(\varphi) \ll x_{t}(\psi)$ for all $t \geq 3 r$. More precisely, $\varphi<\psi$ implies that $x(t, \varphi)<x(t, \psi)$ and $D\left(t, x_{t}(\varphi)\right)<D\left(t, x_{t}(\psi)\right)$ for all $t \geq 2 r$.

Proof. By Lemma 3.1, any solution of (3.1) is defined for all $t \geq 0$. Because of the $r$-periodicity of $x(t, \varphi)$, by assumption (a3) we have

$$
F(t, x(t, \varphi), x(t-r, \varphi))=F(t, x(t, \varphi), x(t, \varphi))=0 .
$$

Therefore, making a change of variables $w(t)=x(t, \psi)-x(t, \varphi)$, we obtain

$$
\frac{d}{d t}[w(t)-b(t) w(t-r)]=F^{*}[t, w(t), w(t-r)],
$$

where

$$
F^{*}(t, u, v)=F(t, u+x(t, \varphi), v+x(t, \varphi)) \text { for }(u, v) \in R^{2},
$$

is $r$-periodic in $t, F^{*}(t, u, u)=0$, and $F^{*}(t, u, v)$ is increasing in $v$ when $(t, u) \in R^{2}$ is fixed. Therefore by Lemma 3.1 (replacing $F$ by $F^{*}$ ), we obtain

$$
\frac{1}{1-b(t)} m(\psi-\varphi) \leq x(t, \psi)-x(t, \varphi) \leq \frac{1}{1-b(t)} M(\psi-\varphi)
$$

and

$$
m(\psi-\varphi) \leq D\left(t, x_{t}(\psi)\right)-D\left(t, x_{t}(\varphi)\right) \leq M(\psi-\varphi)
$$

for $t \geq 0$. This implies conclusion (i).

To prove (ii), we assume that $\varphi\left(\theta_{0}\right)<\psi\left(\theta_{0}\right)$ for a $\theta_{0} \in[-r, 0]$. By (i), $w\left(r+\theta_{0}\right)-b\left(r+\theta_{0}\right) w\left(\theta_{0}\right) \geq 0$. If $w\left(r+\theta_{0}\right)-b\left(r+\theta_{0}\right) w\left(\theta_{0}\right)=0$, then

$$
w\left(r+\theta_{0}\right)=b\left(r+\theta_{0}\right) w\left(\theta_{0}\right)<w\left(\theta_{0}\right)
$$

and thus at $t=r+\theta_{0}$, one has

$$
\begin{aligned}
\frac{d}{d t}[w(t)-b(t) w(t-r)] & =F^{*}[t, w(t), w(t-r)] \\
& =F^{*}\left[r+\theta_{0}, w\left(r+\theta_{0}\right), w\left(\theta_{0}\right)\right] \\
& >0 .
\end{aligned}
$$

Therefore there exists $\varepsilon>0$ such that

$$
w(t)-b(t) w(t-r)>0 \text { for } t \in\left(r+\theta_{0}, r+\theta_{0}+\varepsilon\right] .
$$

For any given constant $T>2 r$, find a constant $L$ by (a5) such that

$$
F^{*}[t, w(t), w(t-r)] \geq-L[w(t)-w(t-r)]
$$

for all $t \in[-r, T]$. Hence we have

$$
\begin{aligned}
\frac{d}{d t}[w(t)-b(t) w(t-r)] & \geq-L[w(t)-w(t-r)] \\
& \geq-L[w(t)-b(t) w(t-r)]
\end{aligned}
$$

from which it follows that

$$
w(t)-b(t) w(t-r) \geq[w(\tau)-b(\tau) w(\tau-r)] e^{-L(t-\tau)}>0
$$


where $\tau$ is either $r+\theta_{0}$ if $w\left(r+\theta_{0}\right)-b\left(r+\theta_{0}\right) w\left(\theta_{0}\right) \neq 0$, or, otherwise, any constant in $\left(r+\theta_{0}, r+\theta_{0}+\varepsilon\right]$. This implies that $w(t)-b(t) w(t-r)>0$ for all $t \geq 2 r$, from which and by $w(t) \geq 0$ for $t \geq-r$ it follows that $w(t)>0$ for all $t \geq 2 r$. This completes the proof.

Likewise, one can prove

Lemma 3.3. Let $x(t, \varphi)$ denote an r-periodic solution of $(3.1)$, and $x(t, \psi)$ be any given solution of (3.1). Then

(i) $\psi \leq \varphi$ implies that $x_{t}(\psi) \leq x_{t}(\varphi)$ for all $t \geq 0$;

(ii) $\psi<\varphi$ implies that $x_{t}(\psi) \ll x_{t}(\varphi)$ for all $t \geq 3 r$. More precisely, $\psi<\varphi$ implies that $x(t, \psi)<x(t, \varphi)$ and $D\left(t, x_{t}(\psi)\right)<D\left(t, x_{t}(\varphi)\right)$ for all $t \geq 2 r$.

Remark 3.1. In the proof of Lemma 3.2, if $x(t, \varphi)$ is not $r$-periodic in $t$, then $w(t)$ satisfies the following equation:

$$
\frac{d}{d t}[w(t)-b(t) w(t-r)]=G^{*}[t, w(t), w(t-r)]
$$

where $G^{*}: \mathbb{R}^{3} \rightarrow \mathbb{R}^{1}$ is defined by

$$
G^{*}(t, u, v)=F(t, u+x(t, \varphi), v+x(t-r, \varphi))-F(t, x(t, \varphi), x(t-r, \varphi)) \text {. }
$$

Since $G^{*}$ does not necessarily satisfy the order relation $G^{*}(t, u, v) \leq 0$ for $(t, u, v) \in \mathbb{R}^{3}$ with $v \leq u$, we cannot apply Lemma 3.1 (replacing $F$ by $G^{*}$ ) to obtain the conclusion (i) of Lemma 3.2. This indicates that our argument cannot be applied to prove the monotonicity and eventually strong monotonicity of the Poincaré map $S: C \rightarrow C$ defined by $S(\varphi)=x_{r}(\varphi)$ for $\varphi \in C$ in the sense of Hirsch [11] and Takáć [16].

Now we are in the position to state our main result.

Theorem 3.1. For any $\varphi \in C$, there exists a constant $k=k(\varphi)$ such that

(i) $\lim _{t \rightarrow \infty}[x(t, \varphi)-k(\varphi) /(1-b(t))]=0$;

(ii) either $x(t, \varphi) \equiv k(\varphi) /(1-b(t))$ for sufficiently large $t$, or for each $n \geq 1$,

$$
\max _{\theta \in[-r, 0]}\left\{x(n r+\theta, \varphi)-\frac{k(\varphi)}{1-b(\theta)}, D\left(n r, x_{n r}(\varphi)\right)-k(\varphi)\right\}>0
$$

and

$$
\min _{\theta \in[-r, 0]}\left\{x(n r+\theta, \varphi)-\frac{k(\varphi)}{1-b(\theta)}, D\left(n r, x_{n r}(\varphi)\right)-k(\varphi)\right\}<0 .
$$

Proof. Consider the Poincare map $S: C \rightarrow C$ defined by $S(\varphi)=x_{r}(\varphi)$ for $\varphi \in C$. Then for each $\varphi \in C$, the set $\left\{S^{n}(\varphi) ; n \geq 0\right\}=\left\{x_{n r}(\varphi) ; n \geq 0\right\}$ is bounded by Lemma 3.1, and thus is relatively compact by the well-known property of neutral equations with stable $D$-operator (see, e.g., [10]). It is easy to prove that a fixed point of $S$ is an $r$-periodic function and the set $E$ of fixed points of $S$ contains a simply ordered arc given by $J: \mathbb{R} \rightarrow C$ defined by

$$
J(\tau)(\theta)=\frac{\tau}{1-b(\theta)} \text { for } \theta \in[-r, 0] \text { and } \tau \in \mathbb{R} .
$$


By Lemmas 3.2 and 3.3, $S$ satisfies assumptions (i) and (ii) of Theorem 2.1.

For any $\varphi \in C$, we define

$$
\alpha(\varphi)=\min \left\{\min _{\theta \in[-r, 0]}[1-b(\theta)] \varphi(\theta), \varphi(0)-b(0) \varphi(-r)\right\}
$$

and

$$
\beta(\varphi)=\max \left\{\max _{\theta \in-[r, 0]}[1-b(\theta)] \varphi(\theta), \varphi(0)-b(0) \varphi(-r)\right\} .
$$

Then

$$
J(\alpha(\varphi))(\theta) \leq \frac{1}{1-b(\theta)}[1-b(\theta)] \varphi(\theta)=\varphi(\theta), \quad \theta \in[-r, 0]
$$

and

$$
D(0, J(\alpha(\varphi)))=\frac{\alpha(\varphi)}{1-b(0)}-b(0) \cdot \frac{\alpha(\varphi)}{1-b(-r)}=\alpha(\varphi) \leq \varphi(0)-b(0) \varphi(-r) .
$$

That is, $J(\alpha(\varphi)) \leq \varphi$. Likewise, $\varphi \leq J(\beta(\varphi))$.

This verifies assumption (iii) of Theorem 2.1. Therefore there exists a constant $k(\varphi)$ such that $S^{n}(\varphi)=x_{n r}(\varphi) \rightarrow J(k(\varphi))$ as $n \rightarrow \infty$. By the continuity of $x_{t}(\varphi)$ with respect to $(t, \varphi) \in \mathbb{R}^{1} \times C$, we obtain $x_{t}(\varphi)(\theta) \rightarrow$ $k(\varphi) /(1-b(t+\theta))$, uniformly for $\theta \in[-r, 0]$, as $t \rightarrow \infty$. This proves (i).

To prove (ii), we assume that $x(t, \varphi)$ does not coincide with $k(\varphi) /(1-b(t))$ for sufficiently large $t$. If

$$
\max _{\theta \in[-r, 0]}\left\{x(n r+\theta, \varphi)-\frac{k(\varphi)}{1-b(\theta)}, D\left(n r, x_{n r}(\varphi)\right)-k(\varphi)\right\} \leq 0
$$

for some $n \geq 1$, then $S^{n}(\varphi)<J(k(\varphi))$. By Lemma 3.3, $S^{n+3}(\varphi) \ll J(k(\varphi))$, i.e.

$$
\frac{k(\varphi)}{1-b(\theta)}-x((n+3) r+\theta, \varphi)>0 \text { for } \theta \in[-r, 0]
$$

and

$$
k(\varphi)>D\left((n+3) r, x_{(n+3) r}(\varphi)\right) .
$$

Therefore we can find a constant $k^{*}>0$ such that $k(\varphi)>k^{*}>\max \left\{\max _{\theta \in[-r, 0]}[1-b(\theta)] x((n+3) r+\theta, \varphi), D\left((n+3) r, x_{(n+3) r}(\varphi)\right)\right\}$.

That is,

$$
S^{n+3}(\varphi) \ll J\left(k^{*}\right) \ll J(k(\varphi)) .
$$

Since $J\left(k^{*}\right) \in E$, it follows from Lemma 3.3 that

$$
S^{n+j}(\varphi) \leq J\left(k^{*}\right) \ll J(k(\varphi)) \text { for } j \geq 3 ;
$$

so $\lim _{n \rightarrow \infty} S^{n}(\varphi) \leq J\left(k^{*}\right) \ll J(k(\varphi))$, a contradiction to $\lim _{n \rightarrow \infty} S^{n}(\varphi)=$ $J(k(\varphi))$. Therefore

$$
\max _{\theta \in[-r, 0]}\left\{x(n r+\theta, \varphi)-\frac{k(\varphi)}{1-b(\theta)}, D\left(n r, x_{n r}(\varphi)\right)-k(\varphi)\right\}>0 .
$$


Likewise, we can prove

$$
\min _{\theta \in[-r, 0]}\left\{x(n r+\theta, \varphi)-\frac{k(\varphi)}{1-b(\theta)}, D\left(n r, x_{n r}(\varphi)\right)-k(\varphi)\right\}<0 .
$$

The proof is completed.

\section{ACKNOWLEDGMENT}

The author wishes to express his gratitude to the referee for valuable comments.

\section{REFERENCES}

1. O. Arino and E. Haourgui, On the asymptotic behavior of solutions of some delay differential systems which have a first integral, J. Math. Anal. Appl. 122 (1987), 36-46.

2. O. Arino and P. Seguier, About the behavior at infinity of solutions of $\dot{x}(t)=$ $f(t-1, x(t-1))-f(t, x(t))$, J. Math. Anal. Appl. 96 (1983), 420-436.

3. F. Atkinson and J. Haddock, Criteria for asymptotic constancy of solutions of functional differential equations, J. Math. Anal. Appl. 91 (1983), 410-423.

4. F. Atkinson, J. Haddock, and O. Staffans, Integral inequalities and exponential convergence of differential equations with bounded delay, Ordinary and Partial Differential Equations (W. Everitt and B. Sleeman, eds.), Lecture Notes in Math., Springer-Verlag, New York, 1982 , pp. 56-68.

5. K. L. Cooke and J. L. Kaplan, A periodicity threshold theorem for epidemic and population growth, Math. Biosci. 31 (1976), 87-104.

6. K. L. Cooke and J. Yorke, Some equations modeling growth process and gonorrhea epidemics, Math. Biosci. 16 (1973), 75-107.

7. I. Györi, Connections between compartmental systems with pipes and integrodifferential equations, Math. Model. 7 (1986), 1215-1238.

8. J. R. Haddock and J. Terjeki, Liapunov-Razumikhin functions and invariance principle for functional differential equations, J. Differential Equations 48 (1983), 95-122.

9. J. R. Haddock, T. Krisztin, and J. Wu, Asymptotic equivalence of neutral equations and infinite delay equations, Nonlinear Anal. TMA 14 (1990), 369-377.

10. J. K. Hale, Theory of functional differential equations, Springer-Verlag, New York, 1977.

11. M. Hirsch, Attractors for discrete-time monotone dynamical systems in strongly ordered spaces, Geometry and Topology, Lecture Notes in Math., vol. 1167, Springer-Verlag, Berlin, Heidelberg, and New York, 1985.

12. M. Hirsch, Stability and convergence in strongly monotone dynamical systems, J. Reine Angew. Math. 383 (1988), 1-53.

13. J. K. Kaplan, M. Sorg, and J. Yorke, Solutions of $\dot{x}(t)=f(x(t), x(t-L))$ have limits when $f$ is an order relation, Nonlinear Anal. TMA 3 (1979), 53-58.

14. H. Smith, Periodic solutions of periodic competitive and cooperative systems, SIAM J. Math. Anal. 17 (1986), 1289-1318.

15. O. J. Staffans, A neutral FDE with stable D-operator is retarded, J. Differential Equations 49 (1983), 208-217.

16. P. Takáč, Convergence to equilibrium on invariant d-hypersurface for strongly increasing discrete-time semigroup, preprint, 1989.

17. J. Wu, Convergence of monotone dynamical systems with minimal equilibria, Proc. Amer. Math. Soc. 106 (1989), 907-911.

Department of Mathematics, University of Alberta, Edmonton, Alberta, Canada T6G 2G1

Current address: Department of Mathematics and Statistics, York University, North York, Ontario, Canada M3J 1P3 